\title{
Monte Carlo simulation of the chemical potential of polymers in an expanded ensemble
}

\author{
Fernando A. Escobedo and Juan J. de Pablo \\ Department of Chemical Engineering, University of Wisconsin-Madison, Madison, Wisconsin 53706-1691
}

(Received 23 January 1995; accepted 9 May 1995)

\begin{abstract}
A new method is proposed for calculation of the chemical potential of macromolecules by computer simulation. Simulations are performed in an expanded ensemble whose states are defined by the length of a tagged molecule of variable size. A configurational-bias sampling and a preweighting scheme are introduced to facilitate transitions between such states. The usefulness of the method is illustrated by calculations of the chemical potential of hard chain molecules over a wide range of densities. The method proposed here is shown to offer significant advantages over other available methods for calculation of chemical potentials, particularly for long chain molecules at high densities. (c) 1995 American Institute of Physics.
\end{abstract}

\section{INTRODUCTION}

Accurate estimation of the chemical potential is essential for study of phase equilibrium in any model system. Calculation of the chemical potential by computer simulation has been the subject of a number of studies. ${ }^{1-10}$ Perhaps the most direct and widely used method for simulation of the chemical potential is the particle insertion method first introduced by Widom. ${ }^{1}$ In this method, a "ghost" particle is momentarily inserted at a random point in the system; the resulting interaction energy $U_{g}$ between the ghost particle and the host fluid is averaged to yield the excess chemical potential, $\mu_{\mathrm{ex}}$, which is given by

$$
\mu_{\mathrm{ex}}=-\frac{1}{\beta} \ln \left\langle\exp \left(-\beta U_{g}\right)\right\rangle,
$$

where $\beta=\left(k_{B} T\right)^{-1}\left(k_{B}\right.$ is Boltzmann's constant), and the brackets denote a canonical ensemble average.

Unfortunately, the particle insertion method has several limitations which preclude its use for many important applications. As the density of the system becomes higher, few random insertions result in low enough interaction energies to make significant contributions to the average appearing in Eq. (1). This problem is more severe in polymeric systems, where upon a random insertion of a whole molecule, steric overlaps become more probable as the chain length increases.

A number of methods have been devised to circumvent this problem. The "inverse-Widom" method ${ }^{2}$ is based on the temporary deletion of a "real" particle from the system; unfortunately, high energy (overlapping) configurations, which contribute significantly to the chemical potential, are not sampled efficiently. The Widom and inverse-Widom methods have actually been combined by Shing and Gubbins ${ }^{2}$ into an efficient algorithm which, for small, spherical molecules, has been shown to be highly effective. ${ }^{3}$

Three important methods have been developed to address the problems encountered in polymeric systems. The continuum configurational-bias (CCB) method ${ }^{4-7}$ extends the range of applicability of Widom's method by allowing the ghost chain molecule to grow, segment by segment, into favorable (low) energy configurations in the host system. The bias introduced in proposing the ghost-molecule configuration is subsequently removed by modifying the interaction energy by a suitable weight. Unfortunately, the CCB approach becomes less effective as the chains become longer (i.e., more than 20 segments long) and as the density of the system becomes higher.

The "chain-increment" method proposed by Kumar and co-workers ${ }^{8-10}$ is based on the observation that, for long chains, the chemical potential associated with "appending" one ghost end segment to a chain becomes chain-length independent. Since the chemical potential of a chain is the sum of the incremental chemical potential associated with a segmental ghost chain growth, these authors approximate the chemical potential of a chain as the sum of identical segmental chemical potentials. A more refined application of the method must necessarily introduce corrections from simulations of shorter ghost chains for which the chemical potential is a function of chain length. This method, however, cannot be applied in its present form to heteronuclear or to branched polymers. Note also that for a system of $n$-mer chains, an exact application of a chain-increment approach would require $n$ sets of independent simulations. ${ }^{10}$

A third method aimed at facilitating the calculation of chemical potentials of polymeric systems has been recently proposed by Wilding and Müller. ${ }^{11}$ Their approach relies on the method of expanded ensembles originally developed by Lyubartsev et al. ${ }^{12}$ and the use of a permanent, penetrable chain which can be gradually coupled or decoupled from the system by tuning an interaction-strength parameter. ${ }^{11,13}$ The method has been reported to provide accurate measures of the chemical potential of long chains at intermediate densities for a lattice system. ${ }^{11}$ Such a method, however, requires the determination of a number of preweighting factors; a process that we have found to be tedious and time consuming.

In this work we present a method that advantageously combines some elements from the three methods aforementioned (CCB, chain-increment, and expanded ensemble methods). The method relies on attempting CCB segmental insertions and deletions on a "tagged" chain. In contrast to Widom's or inverse-Widom's methods, the complete tagged chain need not be grown or deleted at once; instead, a pre- 
selected number of intermediate lengths of the "variable" chain are allowed by defining them as different "states" of an expanded ensemble. Although the method that we propose resembles in some respects the exact chain-increment approach previously mentioned, it requires only a single simulation. Further, it naturally incorporates into the calculation of the chemical potential contributions associated with the segmental deletion process, and it is equally applicable to branched and heteronuclear polymers.

Hard-core systems present a particularly stringent test for the efficiency of simulation techniques aimed at computing the chemical potential. ${ }^{3}$ The method proposed here is applied to hard homopolymer chain molecules of up to 32 segments and packing fractions of up to 0.40 . Some comparative calculations with other methods are also performed and additional discussions are provided to demonstrate the relative advantages of the approach proposed in this work.

\section{THEORY}

\section{A. Expanded ensemble simulations and chemical potential}

Lyubartsev et al. ${ }^{12}$ originally proposed that a single simulation in an expanded ensemble could be used to evaluate the ratio of partition functions of any pair of its subensembles. For the case of canonical ensembles, an expanded ensemble is defined through the following partition function:

$$
\Omega=\sum_{m=1}^{M} Q\left(N, V, \gamma_{m}\right) \exp \left(\psi_{m}\right),
$$

where $Q\left(N, V, \gamma_{m}\right)$ denotes the canonical partition function corresponding to a particular state of the system characterized by the value of a parameter $\gamma_{m}$. To each state corresponds a positive preweighting factor $\psi_{m}$ (whose relevance will be discussed later). A set of $M$ states are selected by assigning to each of them a value of $\gamma$ in the range $\left[\gamma_{1}, \gamma_{M}\right]$. The ultimate goal is to connect smoothly the systems at the two ends of the spectrum: i.e., the reference state $(m=1)$ and the system at the state of interest $(m=M)$.

Lyubartsev and co-workers ${ }^{12}$ were interested in computing the Helmholtz free energy $(A)$ at any given temperature; consequently, they implemented a temperature expanded ensemble by choosing $\gamma_{m}=\beta_{m}$, where $\beta=1 /\left(k_{B} T\right)\left(k_{B}\right.$ is Boltzmann's constant).

In the expanded ensemble formalism, two types of Monte Carlo moves can occur: (1) the usual molecular rearrangements at constant value of $\gamma_{m}$ and (2) trial transitions between neighboring $\gamma$ states. The probability with which each of the $\gamma$ states is visited can be found from

$$
p(m)=p\left(\gamma_{m}\right)=\frac{Q\left(N, V, \gamma_{m}\right)}{\Omega} \exp \left(\psi_{m}\right) .
$$

These probabilities are obtained throughout the course of an expanded ensemble simulation by accumulating a histogram of visits to $\gamma$ states. From Eq. (3) it follows that for any two states $m_{1}$ and $m_{2}$ :

$$
\frac{Q\left(N, V, \gamma_{m_{1}}\right)}{Q\left(N, V, \gamma_{m_{2}}\right)}=\frac{p\left(m_{1}\right) \exp \left(-\psi_{m_{1}}\right)}{p\left(m_{2}\right) \exp \left(-\psi_{m_{2}}\right)} .
$$

Equation (4) is the key relationship for the evaluation of a property that depends on the ratio of two canonical partition functions, such as $A(T)$ (Lyubartsev et al.), ${ }^{12}$ or the chemical potential $\mu$ (Wilding and Müller), ${ }^{11}$ namely,

$$
\exp \left(-\beta \mu_{\mathrm{ex}}\right)=\frac{Q(N+1, V, T)}{Q(N, V, T)} .
$$

If an expanded ensemble is constructed such that the $(N+1)$ th molecule is gradually turned on in going from state 1 (molecule fully decoupled) to state $M$ (molecule fully coupled), from Eqs. (4) and (5) we can write

$$
\beta \mu_{\mathrm{ex}}=-\ln \left[\frac{Q\left(N, V, T, \gamma_{M}\right.}{Q\left(N, V, T, \gamma_{1}\right.}\right]=\ln \left[\frac{p(1)}{p(M)}\right]+\psi_{M}-\psi_{1} .
$$

The preweighting factors $\psi_{m}$ play a critical role in the calculation scheme by encouraging visits to less accessible states, thereby allowing accurate estimates of the probabilities $p(\gamma)$.

\section{B. Penetrable chain method}

Wilding and Müller ${ }^{11}$ considered a system where molecules interact via a short-range attractive potential and excluded-volume restrictions. A penetrable chain experiences the same intramolecular interactions as other molecules in the system. However, the interactions between the penetrable chain and the host system are controlled by a parameter $\lambda$ (defined in the range $[0,1]$ ). The attractive part of the potential is modified by a factor $(1-\lambda)$, while the repulsive part is replaced by an effective potential $K=-(1 / \beta) \ln \lambda$. If $\lambda=0$, the penetrable chain behaves as any other molecule while, for $\lambda=1$, it effectively disappears from the system. The penetrable chain experiences a total repulsive energy given by

$$
E_{\mathrm{rep}}=-\frac{1}{\beta} N_{\mathrm{ov}} \ln \lambda
$$

where $N_{\text {ov }}$ is the total number of overlaps between the penetrable chain and the rest of the molecules.

To cast the method into the expanded ensemble framework, a number $M$ of $\lambda$ states are considered $\left(\lambda_{0}=0\right.$, $\left.\lambda_{M-1}=1\right)$, i.e., $\gamma_{m} \rightarrow \lambda_{M-m}$ in Eq. (2). The acceptance criteria for transition attempts adopted by Wilding and Müller are a straightforward implementation of Metropolis sampling. ${ }^{11,12,14}$ Note, however, that these authors apparently did not consider the asymmetry in the number of neighboring states encountered at both ends of the state spectrum, whose effect in the simulated chemical potential becomes noticeable for small chains.

A uniform distribution for the probability of visiting each state is highly desirable [e.g., $p\left(\lambda_{m}\right)=$ constant], but the corresponding weights cannot be determined a priori. Wilding and Müller ${ }^{11}$ provide a useful iterative scheme to get adequate values for the preweighting factors. In this scheme, a few preliminary simulations are performed where the preweighting factors (initially set to a common value, i.e., 
$\left.\psi_{m}=0\right)$ are successively adjusted [based on the resulting counts of the $p(\lambda)$ histogram] until comparable values of $p(\lambda)$ are attained for each state.

\section{The new method: Expanded variable-length chain simulations}

We use the acronym EVALENCH (expanded variablelength chain method) to distinguish our proposed method for simulation of the chemical potential from that of Wilding and Müller or from the CCB method.

The canonical ensemble partition function for a system of $N$ homopolymer $n$-mer chains and one tagged $m$-mer molecule can be expressed as ${ }^{15}$

$$
\begin{aligned}
Q(N, V, T, m)= & \frac{\Lambda^{-3\left(N+1-\delta_{0, m}\right)}}{\left(N+1-\delta_{0, m}\right) !} \int \exp \left[-\beta U_{N}\left(\mathbf{r}^{N}\right)\right] \\
& \times d \mathbf{r}^{N} \exp \left[-\beta U_{g}\left(\mathbf{r}_{m}\right) d \mathbf{r}_{m}\right],
\end{aligned}
$$

where $U_{g}\left(\mathbf{r}_{m}\right)$ denotes the total interaction energy between the tagged molecule (of coordinates $\mathbf{r}_{m}$ ) and the rest of the system (including the tagged chain's intramolecular interactions), and $U_{N}\left(\mathbf{r}^{N}\right)$ represents the total interaction energy among all other molecules except the tagged molecule $(\Lambda$ is the de Broglie thermal wavelength). It is convenient to define an expanded ensemble through the following partition function:

$$
\Omega=\sum_{k=1}^{M} Q\left(N, V, T, m_{k}\right) \exp \left(\psi_{k}\right),
$$

where $m_{1}=0, m_{M}=n$, and for any other intermediate state, $m_{k}$ is an integer number such that $0<m_{k}<m_{k+1}<n$. In other words, different states of the ensemble correspond to different chain lengths of the tagged chain; for $m_{1}$ we have $Q(N, V, T)$, and for $m_{M}$ we have $Q(N+1, V, T)$ in Eq. (9). For the particular case of a one-segment incremental process we would have $M=n+1$ states and $m_{k}=0,1,2, \ldots, n$.

Transition moves occur between neighboring $m$ states (i.e., $m_{k} \rightarrow m_{k+1}$ or $m_{k} \rightarrow m_{k-1}$ ). To facilitate successful segmental growth attempts, even at high densities, the position of every appended segment $i$ is chosen from a set of $N_{\text {samp }}$ random trial configurations with probability ${ }^{7}$

$$
W_{i}=\frac{w_{i}}{\sum_{j=1}^{N_{\text {samp }} w_{j}},}
$$

where $w_{j}$ is the Boltzmann weight, $\exp \left(-\beta U_{j}\right)$, associated with the $j$ th trial configuration $\left(U_{j}\right.$ is the interaction energy between the appended segment $i$ and the rest of the system, including the existing part of the tagged chain). The probability of proposing a position in which to insert segment $i$, given that one position must be chosen from $N_{\text {samp }}$ possible positions, is

$$
q_{i}=W_{i} / \frac{1}{N_{\mathrm{samp}}} .
$$

We can now implement a Metropolis-type acceptance criteria ${ }^{14,16}$ for a transition attempt from state $m_{y}$ to state $m_{x}$ as follows:

$$
P_{\text {accp }}\left(m_{y} \rightarrow m_{x}\right)=\min \left[1, \frac{T(x \rightarrow y)}{T(y \rightarrow x)} \frac{p(x)}{p(y)}\right],
$$

where $T(x \rightarrow y)$ is the probability of proposing a transition from $x$ to $y$, and $p(x)$ is the probability of observing the system at state $x$. For a canonical expanded ensemble, $p(x)$ can be evaluated from an equation analogous to Eq. (3); for any two snapshots of the system at $x$ and $y$ we have

$$
\frac{p(x)}{p(y)}=\exp \left\{-\beta\left[U_{g}\left(m_{x}\right)-U_{g}\left(m_{y}\right)\right]\right\} \exp \left(\psi_{x}-\psi_{y}\right) .
$$

If $m_{a}>m_{b}$, the transition probabilities $T$ are computed from

$$
\begin{aligned}
& T(b \rightarrow a)=\frac{1}{\text { No. of neighboring states to } b} \prod_{j=m_{b}}^{m_{a}} q_{j}, \\
& T(a \rightarrow b)=\frac{1}{\text { No. of neighboring states to } a} .
\end{aligned}
$$

For linear polymers, the number of neighboring states is always 2 except for states 1 and $M$, in which case it is equal to 1. It is important to note that the acceptance criteria (12) applies whether we are proposing a growth or a deletion of segments. This implies that when proposing a deletion transition, a configurational-bias process must be performed to compute the probability of proposing the hypothetical reverse growing process. Also note that the transition from $m_{1}=0$ to $m_{2}$ [insertion of the first site(s) of the tagged chain] need not be attempted by resorting to a configurational-bias sampling process.

The incremental chemical potential associated to appending $\left(m_{\omega}-m_{\alpha}\right)$ segments to an $m_{\alpha}$-mer tagged chain follows from Eqs. (4) and (6):

$$
\beta \mu_{\mathrm{ex}}\left(m_{\alpha} \rightarrow m_{\omega}\right)=\ln \left[\frac{p\left(m_{\alpha}\right)}{p\left(m_{\omega}\right)}\right]+\psi_{\omega}-\psi_{\alpha} .
$$

The excess chemical potential for the system (insertion of the entire tagged chain) is calculated from Eq. (16) by setting $\alpha=1$ and $\omega=M$.

\section{Discussion of the methods}

A successful application of the penetrable-chain method depends on a suitable choice of the number of $\lambda$ states $(M)$ and their distribution in the range [0,1]. In general, more states are necessary for longer chains and higher densities. However, the optimum choice of $M$ implies a less obvious compromise between the correlation time of the random walk through $\lambda$ states, the acceptance rate of the transition attempts, and the actual achievable counts of $p(\lambda) .{ }^{11}$ The selection of a proper distribution of $\lambda$ states is not a trivial task either. Wilding and Müller recommend that the states be concentrated towards the bound $\lambda=1$ (fully decoupled chain). However, our simulations of hard chain systems in a continuum indicate that, at high densities, a higher concentration of states at both ends of the spectrum (i.e., near $\lambda=1$ and near $\lambda=0$ ) is essential to achieve an acceptable performance and, in some cases, to make the method viable. 
Other shortcomings of the penetrable-chain method are briefly noted.

(1) The preweighting factors from simulations of polymers with different chain lengths (at the same density) need not follow any simple or physically intuitive trends, even if identical $\lambda$ states are adopted for all systems (which is not likely to be a good choice).

(2) The effective repulsive potential adopted by Wilding and Müller [Eq. (7)] applies only to hard-core type potentials, and a different scheme is necessary for a different type of repulsive interaction.

(3) For systems with attractive interactions (short-range square well potential), Wilding and Müller found that a more complex iterative scheme was needed to estimate the preweighting factors.

In contrast to the penetrable chain method, the EVALENCH method leads to a useful relationship between the preweighting factors used for polymer systems of different chain lengths. It follows from Eq. (16) that for a perfectly uniform distribution of $p(m)$, the preweighting factors would directly translate into values of incremental chemical potentials, i.e., $\beta \mu_{\mathrm{ex}}\left(m_{\alpha} \rightarrow m_{\omega}\right)=\psi_{\omega}-\psi_{\alpha}$. For homologous chains at intermediate and high densities, it is known that the insertion probability of an $n_{2}$-mer into an $n_{1}$-mer fluid can be approximated by the insertion probability of an $n_{2}$-mer into an $n_{2}$-mer $\left(n_{1} \geqslant n_{2}\right) \cdot{ }^{17,18}$ Under the assumption of a flat $p(m)$ distribution, we can use the $\Delta \mu$ values obtained for the $n_{2}$-mer at any given density as initial values for the preweighting factors of longer chains, at least for $m \leqslant n_{2}$. Further, for simplicity, we can appeal to the relative independence of the segmental chemical potential and chain length ${ }^{8}$ to generate a full spectrum of preweighting factors for $m_{k}>n_{2}$. In this manner, the information gained from the simulations of a given chain length can be fully exploited for the simulation of longer chains. Using this procedure, we can minimize the number of preliminary simulations required to optimize the values of the preweighting factors for a set of systems of increasing chain lengths. Note that, to be useful, a set of preweighting factors must lead to relatively uniform values of $p(m)$; improved statistics are achievable with preweighting factors that narrow the breadth of $p(m)$ values.

This building up of information characteristic of the EVALENCH method can also be exploited for simulations of polymer blends or solutions of polymers in a solvent. Further, once a suitable growing scheme for the tagged chain has been preset, the EVALENCH method simplifies significantly the calculation of chemical potentials of more complex polymer structures such as copolymers and branched chains. For instance, if a branched chain is grown in two steps, first the main backbone and then the branches, we could estimate the preweighting factors from simulations of shorter single chains.

The penetrable chain method requires a relative larger number of molecular moves between transition attempts to decorrelate the number of overlaps between the host system and the entire excluded volume shell of the tagged chain. In the EVALENCH method, it is mostly the environment in the neighborhood of one end of the chain which needs to be decorrelated after a transition since the other sites of the chain consistently experience conventional interactions for several transition attempt cycles.

Given the ease with which the preweighting factors can be estimated, the choice of an optimum number of states is not too critical for the EVALENCH method. We can arbitrarily choose the number of states in the range $[2, n+1]$ and, in fact, even more than $(n+1)$ states could be considered if segments were appended in stages of increasing strength (i.e., additional penetrable intermediate states). This is likely to be necessary for chemically detailed polymers. On the other hand, it is not efficient to have incremental chain lengths larger than two or three sites, particularly for systems at intermediate or high densities. For very long chains, this implies having numerous transition states which would require very long simulations. Consider, however, that fewer transition states will require more molecular moves per transition attempt to decorrelate the configurational changes generated by more drastic transitions, i.e., fewer states need not translate into shorter simulations to get accurate results of the chemical potential.

\section{SIMULATIONS}

Calculations of chemical potential have been performed for systems of 4-mer, 8-mer, 16-mer, and 32-mer homopolymer hard chains. All chain molecules consist of freely jointed tangent hard spheres. We studied a wide range of densities, from a low value of the packing fraction $\eta=0.10$, consistent with gaslike conditions, up to a value of $\eta=0.40$ (sitenumber density $\rho=0.76$ ) which corresponds to a dense liquidlike fluid.

We also calculated the chemical potential associated with purely intramolecular interactions, $\mu_{\text {intra }}(n)$, for different chain lengths $n$. Such a quantity can be computed by using a particle insertion type method at the limit of zero density (inserting the chain in vacuum). Our reference system is an ideal gas which already includes intramolecular interactions. For any given chain length we must therefore subtract $\mu_{\text {intra }}(n)$ from the chemical potential computed in the simulation in order to get the true excess chemical potential. Note, however, that the chemical potential computed from Wilding and Muller's method does not require a correction for $\mu_{\text {intra }}(n)$ because the fully decoupled tagged chain still experiences an intramolecular potential. Calculation of $\mu_{\text {intra }}(n)$ with the EVALENCH method is particularly simple and requires only one basic simulation for the longest chain of interest; the chemical potential for any shorter chain can be directly calculated from Eq. (16). The results from this calculation yield also a lower bound for the preweighting factors to be used in the calculations at finite densities.

To take advantage of the segmental chemical potentials computed from the shorter chains (as explained in Sec. II), simulations with the EVALENCH method were performed in sequence (i.e., first for the 4-mer and last the 32-mer). For simplicity, we adopted one-segment increments to define the states, i.e. $\left(m_{k+1}-m_{k}\right)=1$. Typically, only one short preliminary simulation was required to get adequate values of the preweighting factors. 


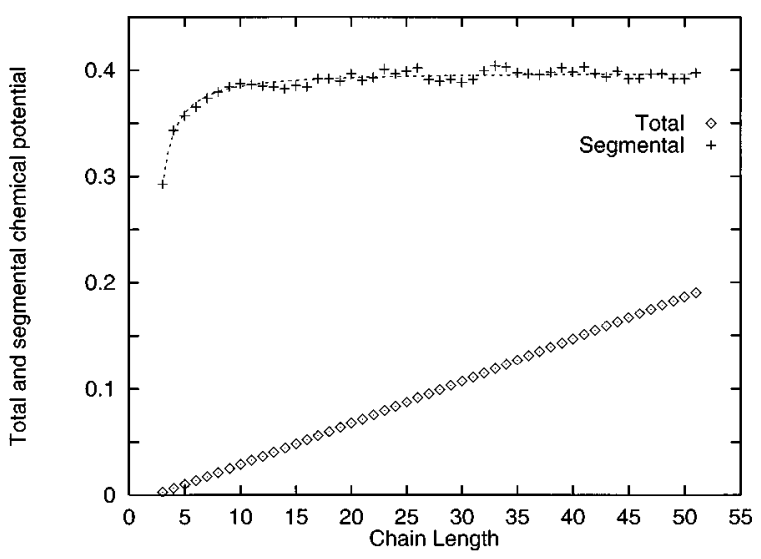

FIG. 1. Zero-density reduced total chemical potential $\left[\beta \mu_{\text {intra }}(n) i \times 10^{-2}\right]$ and segmental increments as a function of chain length for freely jointed hard-sphere chains. The dotted line shows the results from Eq. (17).

The relative frequency of the two types of moves during the expanded ensemble simulation, i.e., molecular rearrangements and tagged chain transitions was controlled by specifying $R_{m / t}$, the ratio of the number of molecular moves (counted on a segmental basis) divided by the number of transition attempts. Enough molecular moves must be attempted between transitions so that the system is properly equilibrated, particularly in the neighborhood of the tagged chain. By keeping an acceptance rate of molecular moves comparable to that of transition attempts (i.e., 30\%), a value of $R_{m / t}$ close to 1 is satisfactory for intermediate densities, and values close to 2 are suitable for the higher densities (note that the number of states is larger for longer chains). Molecular moves were performed using the extended continuum configurational-bias method developed recently by us, ${ }^{19}$ which can effectively produce localized molecular rearrangements of both inner and end sections of the chains. Note also that the configuration of a full size tagged chain can be easily decorrelated by exchanging its identity with that of any other chain in the system.
To verify the accuracy of the results obtained by the EVALENCH method, conventional CCB simulations were performed for the 4-mer, 8-mer, and 16-mer systems at intermediate and low densities. A few simulations using the method of Wilding and Müller ${ }^{11}$ were also performed for the 16-mer system. Since simulations are necessarily performed on finite-size systems, the density of the system must be specified consistently; for all methods, the nominal density of the system was imposed to be that of the system without the ghost or tagged chain in it.

\section{RESULTS AND DISCUSSION}

Figure 1 shows the zero density excess chemical potential for hard chains of different lengths. Figure 1 also shows the incremental chemical potential $\Delta \mu_{\text {intra }}(n)$ as a function of chain length. These results are qualitatively similar to those found by Kumar et al. ${ }^{8}$ for a bead-spring model chain with nonbonded beads interacting through a Lennard-Jones potential. The incremental chemical potential levels off substantially after $n=10$ segments, tending to a limiting value of $\beta \Delta \mu_{\text {intra }}(n) \approx 0.4$ (in reduced units). The continuous curve in Fig. 1 corresponds to the fit

$$
\beta \Delta \mu_{\text {intra }}(n)=0.398\left[1-(2 n-3)^{-6 / 5}\right] \quad n \geqslant 2 .
$$

The smoothed values predicted by this correlation were used to correct the chemical potential at finite densities computed from the CCB method and the EVALENCH method.

In Table I we summarize our results for the 4-mer and 8 -mer hard chains at various packing fractions. It can be seen that the values of chemical potential calculated by the EVALENCH method are fully consistent with those computed by the standard CCB method. In the low density regime $(\eta \leqslant 0.2)$, it appears that a high accuracy is more easily achieved with the CCB method where the rate of successful insertions is relatively high. However, for the 8-mer system at $\eta>0.30$ the CCB method becomes significantly less effi-

TABLE I. Simulation results for 4-mer and 8-mer hard-sphere chains. The number of MC steps per molecule is $0.75 \times 10^{6}$ for the 4 -mers and $1.5 \times 10^{6}$ for the 8 -mers. The $\beta \mu_{\mathrm{ex}}$ from simulation have been corrected by $\beta \mu_{\text {intra }}=0.63$ and 2.12 for the 4-mer and 8-mer systems, respectively.

\begin{tabular}{|c|c|c|c|c|c|c|}
\hline$\eta$ & No. chains & $R_{m / t}$ & $\begin{array}{c}N_{\text {samp }} \\
\text { per segment }\end{array}$ & $\begin{array}{c}\beta \mu_{\mathrm{ex}} \\
\text { EVALENCH }\end{array}$ & $\begin{array}{l}\beta \mu_{\mathrm{ex}} \\
\mathrm{CCB}\end{array}$ & $\begin{array}{c}\beta \mu_{\mathrm{ex}} \\
\text { EOS integ }\end{array}$ \\
\hline \multicolumn{7}{|c|}{ 4-mers } \\
\hline 0.10 & 50 & 1 & 4 & $2.13 \pm 0.06$ & $2.08 \pm 0.002$ & 2.06 \\
\hline 0.20 & 75 & 1.5 & 6 & $5.94 \pm 0.07$ & $5.94 \pm 0.007$ & 5.86 \\
\hline 0.25 & 75 & 1.5 & 10 & $8.98 \pm 0.06$ & $8.96 \pm 0.014$ & 8.84 \\
\hline 0.30 & 100 & 2 & 15 & $13.05 \pm 0.08$ & $13.05 \pm 0.03$ & 12.91 \\
\hline 0.35 & 100 & 2 & 24 & $18.85 \pm 0.15$ & $18.77 \pm 0.08$ & 18.52 \\
\hline 0.40 & 125 & 2 & 32 & $26.8 \pm 0.3$ & $27.2 \pm 0.6$ & 26.4 \\
\hline \multicolumn{7}{|c|}{ 8-mers } \\
\hline 0.10 & 50 & 1 & 4 & $3.29 \pm 0.16$ & $3.32 \pm 0.02$ & 3.31 \\
\hline 0.20 & 75 & 1.5 & 6 & $10.14 \pm 0.23$ & $10.22 \pm 0.05$ & 10.02 \\
\hline 0.25 & 75 & 1.5 & 10 & $15.64 \pm 0.28$ & $15.73 \pm 0.10$ & 15.48 \\
\hline 0.30 & 100 & 2 & 15 & $23.43 \pm 0.40$ & $23.44 \pm 0.4$ & 23.1 \\
\hline 0.35 & 100 & 2 & 24 & $34.2 \pm 0.40$ & $34.8 \pm 1.7$ & 33.8 \\
\hline 0.40 & 100 & 2 & 32 & $49.5 \pm 0.60$ & & 49.0 \\
\hline
\end{tabular}


TABLE II. Simulation Results for 16-mer hard-sphere chains. For the EVALENCH method and the Wilding and Müller method (WM): The number of MC steps per molecule was $3.0 \times 10^{6}$. The $\beta \mu_{\mathrm{ex}}$ computed by the EVALENCH method and the CCB method have been corrected by $\beta \mu_{\text {intra }}=5.22$.

\begin{tabular}{|c|c|c|c|c|c|c|}
\hline$\eta$ & No. chains & $\begin{array}{c}R_{m / t} \\
\text { EVALENCH }\end{array}$ & $\begin{array}{l}R_{m / t} \\
\mathrm{WM}\end{array}$ & $\begin{array}{c}\beta \mu_{\mathrm{ex}} \\
\text { EVALENCH }\end{array}$ & $\begin{array}{l}\beta \mu_{\mathrm{ex}} \\
\mathrm{WM}\end{array}$ & $\begin{array}{l}\beta \mu_{\mathrm{ex}} \\
\mathrm{CCB}\end{array}$ \\
\hline 0.10 & 30 & 1 & 1.5 & $5.82 \pm 0.3$ & $5.83 \pm 0.2$ & $5.78 \pm 0.03$ \\
\hline 0.20 & 60 & 1.5 & 2 & $18.5 \pm 0.3$ & $18.7 \pm 0.3$ & $18.6 \pm 0.12$ \\
\hline 0.25 & 75 & 1.5 & 2.5 & $29.1 \pm 0.4$ & $29.3 \pm 0.4$ & $29.1 \pm 0.4$ \\
\hline 0.30 & 90 & 2 & 3 & $44.0 \pm 0.4$ & $44.2 \pm 0.5$ & $44.2 \pm 1.0$ \\
\hline 0.35 & 105 & 2 & 3 & $65.0 \pm 0.5$ & $64.8 \pm 0.7$ & \\
\hline 0.40 & 120 & 2 & 3.5 & $94.7 \pm 2.0$ & $93.2 \pm 4.0$ & \\
\hline
\end{tabular}

cient; very low insertion rates prevail and large values of $N_{\text {samp }}(>50)$ must be used. For 8-mers, the CCB method becomes impractical for $\eta \geqslant 0.40$.

The last column in Table I gives the results found by integration of equations of state that we fitted to computer simulation PV data reported by Chang and Sandler. ${ }^{20,21}$ Although these equation-of-state results are consistent with our simulations, simulated chemical potentials are consistently slightly high. Aside from some inaccuracies in the equation of state, these differences are due to finite-size effects on the simulated chemical potential which are expected to cause an apparent larger value of $\mu_{\mathrm{ex}} \cdot{ }^{11}$ Since the presence of the tagged molecule increases the nominal site density of a finite-size system, larger relative deviations are observed for systems with fewer molecules (in our case at low $\eta$ ). These deviations are inherent to any simulation method and are usually small for a properly chosen system size; for the systems studied here the average relative error is $1.7 \%$.

In Table II we present results for the 16-mer system. For such a system the CCB method becomes impractical for $\eta>0.30$. In implementing Wilding and Müller's method we adopted $12 \lambda$ states in all cases (no attempt was made to find an optimal number of states and the value chosen is likely to be rather large for low $\eta$ and somewhat small for high $\eta$ ). The $\lambda$ values adopted are the result of a preliminary experimentation at $\eta=0.30$ only. The preweighting factors for the different $\lambda$ states and packing fractions are given in Table III. Initially we followed Wilding and Müller's recommendation of concentrating states towards the $\lambda=1$ bound. We found, however, that counts in the $\lambda=0$ limit (chain fully coupled) were not practically achievable in dense systems unless several of the states were also concentrated near this limit. Further, given the large noise in the results, it would appear that even more states should be considered around $\lambda=0$ for $\eta=0.40$.

Results for the 32-mer system are presented in Table IV. No attempt was even made to use either the CCB method or Wilding and Müller's method. Extrapolating from previous results it would appear that the applicability of these two methods to this system (at intermediate and high densities) would be limited.

Figures 2 and 3 show the incremental chemical potential for the different systems at various densities as a function of chain length. All the curves follow a similar trend: $\Delta \mu_{\mathrm{ex}}$ is the largest for the first site inserted, it decreases monotonically for subsequent sites, and it levels off considerably by the third or fourth site. As expected, the incremental chemical potential is larger at higher densities; once a big enough void has been found to accommodate the first site of a chain, the likelihood of finding additional free volume nearby is favored by steric effects and, consequently, the second site is appended more easily than the first, the third site more easily than the second, and so on. After a few sites, this decreasing trend quickly dampens, as the energy environment available to a site and the short range site-site correlations for successive sites become uniform, thereby resulting in almost identical $\Delta \mu_{\mathrm{ex}}$ values. A subtle increasing trend in $\Delta \mu_{\mathrm{ex}}$ is occasionally observed; this might reflect that the local site-

TABLE III. Preweighting factors for simulations for 16-mer hard-sphere chains using Wilding and Müller's method.

\begin{tabular}{ccccccc}
\hline \hline$\lambda_{m}$ & $\eta=0.10$ & $\eta=0.20$ & $\eta=0.25$ & $\eta=0.30$ & $\eta=0.35$ & $\eta=0.40$ \\
\hline 1.00 & 0.00 & 0.00 & 0.00 & 0.00 & 0.00 & 0.00 \\
0.97 & 0.39 & 0.75 & 0.97 & 1.16 & 1.37 & 1.55 \\
0.92 & 1.00 & 2.0 & 2.6 & 3.1 & 3.7 & 4.2 \\
0.85 & 1.75 & 3.8 & 4.9 & 6.0 & 7.1 & 8.1 \\
0.75 & 2.66 & 6.2 & 8.2 & 10.2 & 12.2 & 14.0 \\
0.62 & 3.62 & 9.2 & 12.6 & 15.9 & 19.3 & 22.5 \\
0.45 & 4.50 & 12.6 & 18.0 & 23.9 & 29.8 & 35.4 \\
0.33 & 4.95 & 14.6 & 21.6 & 29.6 & 38.1 & 46.3 \\
0.20 & 5.35 & 16.4 & 24.9 & 35.8 & 48.2 & 60.7 \\
0.10 & 5.61 & 17.6 & 27.2 & 40.1 & 56.4 & 74.9 \\
0.05 & 5.72 & 18.2 & 28.3 & 42.2 & 60.6 & 83.6 \\
$10^{-6}$ & 5.83 & 18.7 & 29.3 & 44.2 & 64.7 & 92.1 \\
\hline \hline
\end{tabular}


TABLE IV. Simulation results for 32-mer hard-sphere chains. For both methods the number of MC steps per molecule was $6 \times 10^{6}$ and the $\beta \mu_{\mathrm{ex}}$ values have been corrected by $\beta \mu_{\text {intra }}$ (=11.52 for the whole chain).

\begin{tabular}{ccccccc}
\hline \hline & & & & $\beta$ & $\beta \mu_{\mathrm{ex}}$ & $\beta \mu_{\mathrm{ex}}$ \\
$\eta$ & No. chains & $\beta \mu_{\mathrm{ex}}(7)$ & $\beta \Delta \mu_{\mathrm{ex}}(7)$ & $\beta \Delta \mu_{\mathrm{ex}}(32)$ & EVALENCH & \begin{tabular}{c} 
Approx. Method \\
\hline 0.10
\end{tabular} \\
0.20 & 55 & 2.68 & 0.32 & 0.305 & $10.6 \pm 0.3$ & 10.5 \\
0.25 & 55 & 13.1 & 1.08 & 1.06 & $35.4 \pm 0.4$ & 35.1 \\
0.30 & 100 & 19.7 & 2.62 & 1.69 & $56.4 \pm 0.5$ & 55.7 \\
0.35 & 100 & 28.8 & 3.90 & 2.59 & $85.3 \pm 0.5$ & 84.8 \\
0.40 & 100 & 41.9 & 5.72 & 5.75 & $126.8 \pm 1.2$ & 125.7 \\
\hline \hline
\end{tabular}

density changes associated with the chain growth process are not fully decorrelated between transitions, or that such changes have a significant effect on the overall density of the system (finite-size effects).

As already exploited for estimation of the preweighting factors, it is observed in Figs. 2 and 3 that the incremental chemical potential at constant density does not differ appreciably for systems of different chain length. This behavior was anticipated based on heuristic arguments and has been used for development of equations of state for hard chains. ${ }^{17,18}$ A more detailed analysis of the correspondence between the behavior of the chemical potential and equations of state can be found elsewhere. ${ }^{21}$

Although the method proposed here is essentially exact (no approximations are invoked), in practice it might also be useful to have alternative, albeit approximate methods which retain to a large extent the accuracy of the EVALENCH method. This is particularly true for dense systems of very long chains (i.e., $n \geqslant 100$ ), where simulations could become prohibitively long. The observation that the segmental chemical potential becomes essentially constant after the first few sites is not restricted to hard chains ${ }^{8}$ and, as mentioned earlier, it is exploited in the "chain increment method." For a system of $n$-mers, a prescription that combines the EVALENCH method with the relative insensitivity of $\Delta \mu_{\mathrm{ex}}$ to chain length can be implemented as follows.

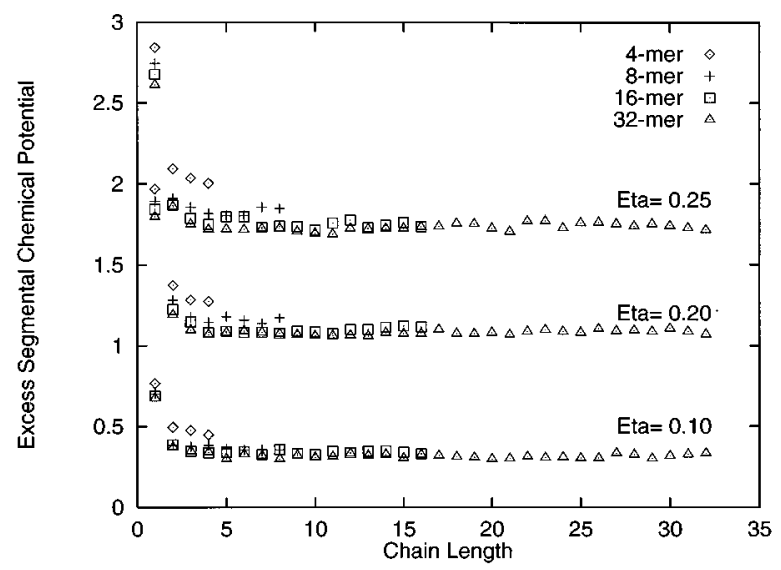

FIG. 2. Segmental excess chemical potential in reduced units, $\beta \Delta \mu_{\mathrm{ex}}$, for freely jointed hard-sphere chains of different lengths at low densities $(\eta$ $=0.1,0.2,0.25)$. The symbols show results for chains of different lengths obtained with the EVALENCH method.
(1) Choose a chain size $n_{1}$ such that $n_{1} \ll n$. This size should be long enough to absorb the range in which $\Delta \mu_{\mathrm{ex}}$ is significantly sensitive to chain length. A suitable choice of $n_{1}$ will depend on the characteristics of the system under study.

(2) Tag two chains, $A$ and $B$. Each state is now specified by the length of both chains $A$ and $B,\left(n_{a}, n_{b}\right)$. Consider the states: $1=(n-1,0) ; \quad 2=(n, 0) ; \quad 3=(n, 1) ; \ldots, M-1$ $=\left(n, n_{1}-1\right), M=\left(n, n_{1}\right)$.

(3) Perform a simulation (for the $n$-mer system) using the EVALENCH method. Tagged molecule $A$ provides the incremental chemical potential for appending an end site, $\Delta \mu_{\mathrm{ex}}(n)$. From tagged chain $B$ we can obtain accurately $\mu_{\mathrm{ex}}\left(n_{1}\right)$ and the incremental chemical potential $\Delta \mu_{\mathrm{ex}}\left(n_{1}\right)$ corresponding to the $n_{1}$ th site appended.

(4) Assume a linear dependency of $\Delta \mu_{\mathrm{ex}}(k)$ with chain length from $k=n_{1}$ to $k=n$, e.g.,

$$
\Delta \mu_{\mathrm{ex}}(k)=\Delta \mu_{\mathrm{ex}}\left(n_{1}\right)+\frac{k-n_{1}}{n-n_{1}}\left[\Delta \mu_{\mathrm{ex}}(n)-\Delta \mu_{\mathrm{ex}}\left(n_{1}\right)\right] .
$$

Using this approximation the chemical potential for the system is given by

$$
\begin{aligned}
\mu_{\mathrm{ex}}(n)= & \mu_{\mathrm{ex}}\left(n_{1}\right)-\Delta \mu_{\mathrm{ex}}\left(n_{1}\right)+\frac{1}{2}\left(n-n_{1}+1\right) \\
& \times\left[\Delta \mu_{\mathrm{ex}}\left(n_{1}\right)+\Delta \mu_{\mathrm{ex}}(n)\right] .
\end{aligned}
$$

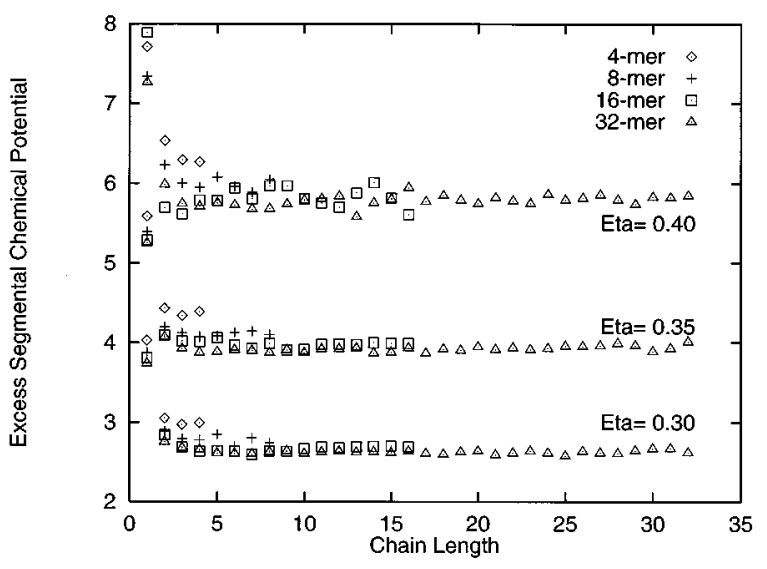

FIG. 3. Segmental excess chemical potential in reduced units, $\beta \Delta \mu_{\mathrm{ex}}$, for freely-jointed hard-sphere chains of different lengths at intermediate-high densities $(\eta=0.30,0.35,0.40)$. The symbols show results for chains of different lengths obtained with the EVALENCH method. 
This simulation should be shorter than the full size simulation by a factor of roughly $\left(n_{1}+1\right) / n$. The computer time savings associated could be substantial in some cases. The last column of Table IV shows the results based on this scheme; for this application $n=32$ and we used $n_{1}=7$. The loss of accuracy is not significant when compared to the statistical error in the results.

Variations of the approximate method can also be implemented (for instance sampling intermediate points of $\Delta \mu_{\mathrm{ex}}$, etc.) and adapted to more complex polymer structures.

\section{CONCLUSIONS}

A new method for calculation of the chemical potential of polymeric systems has been presented. The EVALENCH method combines elements of the CCB approach and the simulation of expanded ensembles to yield a robust, efficient method. A tagged chain visits a number of different chain lengths (ensemble states), thereby generating incremental chemical potentials throughout a simulation. This information in turn facilitates the estimation of the preweighting factors needed for the simulation of similar molecular models of longer chains.

The method has been applied to systems of tangent hard sphere polymers of various lengths at low and high densities. A comparative application of available methods shows that the EVALENCH method overcomes some of the shortcomings of the penetrable chain method, particularly regarding the selection of transition states and estimation of preweighting factors. The EVALENCH method is applicable to ranges of chain lengths and densities where the conventional CCB method becomes impractical. As opposed to the approximate chain-increment method of Kumar et al., ${ }^{8}$ the EVALENCH method is essentially exact and is equally applicable to heteronuclear and branched polymers.

The behavior of the segmental chemical potential has been analyzed for the hard systems studied in this work. The general features of this behavior support the implementation of inexpensive, approximate methods for calculation of the chemical potential of very long chains. An example of such a method has been suggested.
A number of variations of the method presented here are being developed. In particular, simulations of branched polymers are underway. The method provides not only an alternative to the Gibbs ensemble simulations for the study of phase equilibrium of polymeric systems ${ }^{22}$ (in cases where the latter method is inefficient) but it could also be combined with it by developing an expanded Gibbs ensemble.

\section{ACKNOWLEDGMENTS}

The authors gratefully acknowledge the financial support of the National Science Foundation and from the industrial sponsors of the University of Wisconsin Polymerization Reaction Engineering Laboratory. We are also grateful to the Petroleum Research Fund of the American Chemical Society.

${ }^{1}$ B. Widom, J. Chem. Phys. 39, 2808 (1963).

${ }^{2}$ K. S. Shing and K. E. Gubbins, Mol. Phys. 43, 717 (1982).

${ }^{3}$ M. P. Allen and D. J. Tildesley, Computer Simulation of Liquids (Clarendon, Oxford, 1987).

${ }^{4}$ H. Meirovitch, Phys. Rev. A 32, 3699 (1985).

${ }^{5}$ J. I. Siepmann, Mol. Phys. 70, 1145 (1990).

${ }^{6}$ D. Frenkel and B. Smith, Mol. Phys. 75, 983 (1992).

${ }^{7}$ J. J. de Pablo, M. Laso, and U. W. Suter, J. Chem. Phys. 96, 6157 (1992).

${ }^{8}$ S. K. Kumar, I. Szleifer, and A. Z. Panagiotopoulos, Phys. Rev. Lett. 66, 2935 (1991)

${ }^{9}$ S. K. Kumar, J. Chem. Phys. 97, 3550 (1992).

${ }^{10}$ I. Szleifer and A. Z. Panagiotopoulos, J. Chem. Phys. 97, 6666 (1992).

${ }^{11}$ N. B. Wilding and M. Muller, J. Chem. Phys. 101, 4324 (1994).

${ }^{12}$ A. P. Lyubartsev, A. A. Martsinovski, S. V. Shevkunov, and P. N. Vorontsov-Velyaminov, J. Chem. Phys. 96, 1776 (1992).

${ }^{13}$ M. Muller and W. Paul, J. Chem. Phys. 100, 719 (1994).

${ }^{14}$ N. Metropolis, A. W. Rosenbluth, M. N. Rosenbluth, A. H. Teller, and E. Teller, J. Chem. Phys. 21, 1087 (1953).

${ }^{15}$ J. P. Hansen and I. R. McDonald, Theory of Simple Liquids, 2nd ed. (Academic, New York, 1986).

${ }^{16}$ M. H. Kalos and P. A. Whitlock, Monte Carlo Methods (Wiley, New York, 1986), Vol. I.

${ }^{17}$ R. Dickman and C. K. Hall, J. Chem. Phys. 85, 4108 (1986).

${ }^{18}$ K. G. Honnell and C. K. Hall, J. Chem. Phys. 90, 1841 (1989).

${ }^{19}$ F. Escobedo and J. J. de Pablo, J. Chem. Phys. 102, 2636 (1995).

${ }^{20}$ J. Chang and S. I. Sandler, Chem. Eng. Sci. 49, 2777 (1994).

${ }^{21}$ F. Escobedo and J. de Pablo, J. Chem. Phys. 102, 1946 (1995).

${ }^{22}$ M. Laso, J. J. de Pablo, and U. W. Suter, J. Chem. Phys. 97, 2817 (1992). 